\title{
Insulin renders diabetic rats resistant to acute ischemic stroke by arresting nitric oxide reaction with superoxide to form peroxynitrite
}

\author{
Li-Man Hung ${ }^{1}$, Jiung-Pang Huang ${ }^{1}$, Jiuan-Miaw Liao ${ }^{2}$, Meng-Hsuan Yang ${ }^{1}$, Dai-Er Li ${ }^{1}$ Yuan-Ji Day ${ }^{3}$ \\ and Shiang-Suo Huang ${ }^{4^{*}}$
}

\begin{abstract}
Background: The functions of free radicals on the effects of insulin that result in protection against cerebral ischemic insult in diabetes remain undefined. This present study aims to explain the contradiction among nitric oxide (NO)/superoxide/peroxynitrite of insulin in amelioration of focal cerebral ischemia-reperfusion (FC I/R) injury in streptozotocin (STZ)-diabetic rats and to delineate the underlying mechanisms. Long-Evans male rats were divided into three groups (age-matched controls, diabetic, and diabetic treated with insulin) with or without being subjected to FC I/R injury.

Results: Hyperglycemia exacerbated microvascular functions, increased cerebral NO production, and aggravated FC I/R-induced cerebral infarction and neurological deficits. Parallel with hypoglycemic effects, insulin improved microvascular functions and attenuated FC I/R injury in STZ-diabetic rats. Diabetes decreased the efficacy of NO and superoxide production, but NO and superoxide easily formed peroxynitrite in diabetic rats after FC I/R injury. Insulin treatment significantly rescued the phenomenon.
\end{abstract}

Conclusions: These results suggest that insulin renders diabetic rats resistant to acute ischemic stroke by arresting NO reaction with superoxide to form peroxynitrite.

Keywords: Diabetes, Ischemic stroke, Insulin, Nitric oxide, Superoxide, Peroxynitrite

\section{Background}

The principal mediator of cerebral injury secondary to ischemic stroke is oxidative stress [1]. Oxidative stressmediated cerebral injury is the consequence of an imbalance between free radical generation and elimination due to increased reactive oxygen and nitrogen species generation and inadequate antioxidant defense [1]. Central nervous system cells are more vulnerable to oxidative stress given their inherent higher oxidative metabolism and less antioxidant enzymes and higher membranous fatty acid content. During ischemic stroke, the free radicals concentration rises from normal low levels to a peak point during reperfusion, possibly underlying apoptosis or cellular necrosis. Malinski et al. found that the basal nitric

\footnotetext{
* Correspondence: sshuang@csmu.edu.tw

${ }^{4}$ Department of Pharmacology and Institute of Medicine, Chung Shan Medical University and Department of Pharmacy, Chung Shan Medical University Hospital, No.110, Sec. 1, Jianguo N. Rd, Taichung City 402, Taiwan Full list of author information is available at the end of the article
}

oxide (NO) levels are in the nanomolar range, nevertheless, the amount rapidly increases to micromolar levels following cerebral ischemia [1,2]. The cerebral ischemia reperfusion injury can also increase superoxide production [3]. In addition, increasing evidence in both experimental and clinical studies also suggests that oxidative stress is critical in the pathogenesis of both types of diabetes mellitus [4]. However, studies of diabetic NO levels have yielded conflicting results. Some investigators have demonstrated impaired NO production in diabetes [5,6]. By contrast, other studies have observed increased $\mathrm{NO}$ in diabetes [7] or no change in NO level in the diabetic state [8].

In the basic studies, insulin treatment protects neurological function following cerebral ischemia in hyperglycemic rats $[9,10]$. However, the roles of free radicals on the effects of insulin that result in protection against cerebral ischemic insult in diabetes remain undefined. The primary aim of the present study is to resolve the 
discrepancy of expression of $\mathrm{NO}$ in diabetes. The secondary aim is to explore the possible mechanisms (with specific focus on $\mathrm{NO}$ /superoxide/peroxynitrite) of insulin in amelioration of focal cerebral ischemia-reperfusion (FC I/R) injury in streptozotocin (STZ)-diabetic rats.

\section{Methods}

\section{Experimental animals}

Healthy adult male Long-Evans rats (National Lab. Animal Breeding and Research Center) were used throughout this study and were maintained in the Animal Center of the Chang Gung University, at an ambient temperature of $25 \pm 1^{\circ} \mathrm{C}$ and a light-dark period of $12 \mathrm{~h}$. The animals were fed with normal chow and water. This investigation abides by the rules in the Guide for the Care and Use of Laboratory Animals published by the US National Institutes of Health (NIH publication no. 85-23, revised 1996).

\section{Induction of STZ-diabetic animal model}

Diabetes was induced according to the previous-described protocol [11]. The experimental animals were randomly divided into two groups: (1) age-matched non-diabetic control (Con) and (2) STZ-induced diabetic rats (DM). The animals were then injected with a single intravenous injection of vehicle (normal saline) or freshly prepared STZ $(65 \mathrm{mg} / \mathrm{kg})$. The STZ-diabetic animals were further randomly divided into two groups: (1) DM and (2) diabetic rats treated with insulin (DMI; Monotard ${ }^{\circ} \mathrm{HM}$; Novo Nordisk, Bagsvaerd, Denmark).STZ-diabetic rats were subcutaneously injected with insulin (4 IU/rat/day) for 1 week (at 8 to 9 AM from days 15 to 21 of STZ injection). The animals were subjected to FC I/R injury at $8 \mathrm{AM}$ on day 22 .

\section{Surgical procedure and analysis of infarct volume}

Focal ischemic infarcts were carried out by operation as reported previously with some modifications [12]. In brief, rats weighing between $250 \mathrm{~g}$ and $280 \mathrm{~g}$ were anesthetized with chlorohydrate $(4.5 \mathrm{mg} / \mathrm{kg}$, intraperitoneal injection) after $24 \mathrm{~h}$ of the last vehicle (normal saline) or insulin treatment. Rectal temperature was monitored and kept constant $\left(37 \pm 0.5^{\circ} \mathrm{C}\right)$ during the surgical procedure and recovery period. The animals were performed craniotomy at the junction of the zygoma and squamosal bone using a drill (Dremel Multipro + 5395, Dremel com. USA) and the dura was opened with fine forceps using a dissecting microscope (OPMI-1, ZISS, Germany). The right middle cerebral artery (MCA) was ligated with 10-0 monofilament nylon ties and both common carotid arteries were then occluded by microaneurysm clips for $1 \mathrm{~h}$. After removing the clips, return of blood flow was visualized in both common carotid arteries. However, the right MCA ligature was left in place permanently [13].
Animals were sacrificed by rapid decapitation under deep anesthesia. Brains were rapidly removed and sliced into 2-mm thick coronal sections using a brain matrix slicer (JACOBOWITZ Systems, Zivic-Miller Laboratories INC, Allison park, USA) and stained with 2,3,5-triphenyltetrazolium chloride (TTC; 2\%; Sigma-Aldrich, USA) at $37^{\circ} \mathrm{C}$ and kept in the dark for $30 \mathrm{~min}$, followed by fixation with $10 \%$ formalin at room temperature overnight. The infarcted tissue, clearly visible by a lack of TTC staining [14], was outlined on the posterior surface of each slice using an image analyzer (color image scanner, EPSON GT-9000), connected to an image analysis system (AIS software, Imaging research INC, Canada) run on a personal computer, AMD K6-2 3D 400. Infarct volume was obtained as the sum of infarct area per slice multiplied by slice thickness. The infarct volume was expressed as volume $\left(\mathrm{mm}^{3}\right)$ of the whole brain. Both the surgeon and image analyzer operator were blinded to the treatment of each animal.

\section{Assessment of neurological functions}

The modified Bederson score [15] was used $24 \mathrm{~h}$ after FC I/R injury to determine global neurological function based on the following scoring system: 0, no deficit; 1 , forelimb flexion; 2, decreased resistance to lateral push; 3, unidirectional circling; 4, longitudinal spinning; and 5, no movement.

\section{Biochemical analysis}

Blood was collected from the tail vein for biochemical measurements in experimental rats. Plasma was used for the measurements of total cholesterol and triglyceride (Randox reagent kits, Randox Laboratories LTD. Antrim, United Kingdom). Insulin was measured using a commercial available kit provided by Mercodia (rat insulin ELISA kit, Uppsala, Sweden). Blood glucose levels were determined by the glucose oxidase method (ChemWell ${ }^{\circ}$ 2910 Automated EIA and Chemistry Analyzer Spectra, GMI Inc., USA). The NO/ozone chemiluminescence technique (NOA 280; Sievers Instruments; Boulder, CO, USA) was employed for measuring cerebral NO levels. The deproteinized tissue samples were isolated from brain in accordance to previously published procedures with slight modifications [16]. Superoxide production was measured from the cerebrum by lucigenin-enhanced chemiluminescence, using previously described and validated methods [17]. Peroxynitrite production from the cerebrum of different groups of rats was measured by the luminol-enhanced chemiluminescence method as described previously [18].

\section{Intravital microscopic observation of cremasteric microciculation}

The cremaster muscle was prepared for intravital microscopy following methods reported previously [19]. 
Briefly, animals were anesthetized with pentobarbital (65 mg/kg, i.p.) and surgical exposure of the cremaster muscle. The cremasteric microcirculation was visualized using an intravital microscope (Nikon Measurescope MM-22, Tokyo, Japan). A digital camera (Sony DXC-750 MD, Tokyo, Japan) was adapted between intravital microscope and a videocassette recorder (Sony SVO-9600, Tokyo, Japan) and project the images onto a calibrated monitor (Sony). To minimize variability, four to six venules were selected in each trial and the same section of each venule was observed throughout the experiment. The number of rolling, adherent (attached to the vessel wall for at least $20 \mathrm{~s}$ ), and transmigrating leukocytes were counted per region off-line during video playback analysis [20]. Both the surgeon and image analyzer operator were blinded to the treatment of each animal.

\section{Statistics}

Data are expressed as mean \pm standard error of mean (SEM). Statistical analysis was performed by one-way analysis of variance for combined data and followed by Bonferroni's tests. $\mathrm{P}<0.05$ was considered statistically significant.

\section{Experimental animals}

Male Long-Evans rats (age 8 weeks) were purchased from National Lab. Animal Breeding and Research Center (Taipei, Taiwan). The rats were maintained in a climate controlled facility on a 12-h light/12-h dark cycle with free access to water and food. All experimental procedures were conducted in accordance with the Guide for Care and Use of Laboratory Animals and approved by the Institutional Animal Care and Use Committee of the Chang Gung University at Taiwan.

\section{Results}

General characteristics in Non-diabetic control, STZ-diabetic, and insulin-treated diabetic rats

Animals treated with STZ resulted in consistent hyperglycemia and hypoinsulinemia that persisted over the three-week period. At day 21 of the experiment, one week after vehicle or insulin treatment, plasma glucose and insulin levels in diabetic rats were $460 \pm 15 \mathrm{mg} / \mathrm{dl}$ and $0.56 \pm 0.07 \mu \mathrm{g} / \mathrm{l}$ compared with values of $125 \pm 5 \mathrm{mg} / \mathrm{dl}$ and $2.58 \pm 0.36 \mu \mathrm{g} / \mathrm{l}$ in age-matched non-diabetic controls, respectively $(P<0.001$ and $P<0.05$; Table 1$)$. As expected, diabetic rats had lower body weights compared with agematched controls $(292 \pm 11$ vs. $391 \pm 7$ g, $P<0.001$; Table 1). By contrast, insulin treatment (4 IU/rat/day) for 1 week significantly attenuated diabetes-induced body weight loss $(329 \pm 14$ vs. $292 \pm 11 \mathrm{~g}, P<0.05)$, hyperglycemia $(153 \pm 10$ vs. $460 \pm 15 \mathrm{mg} / \mathrm{dl}, P<0.001)$, and hypoinsulinemia $(2.04 \pm 0.55$ vs. $0.56 \pm 0.07 \mu \mathrm{g} / \mathrm{l}, P<0.05)$ (Table 1).
Table 1 Laboratory characteristics in age-matched nondiabetic control (Con), diabetic (DM), and insulin-treated diabetic (DMI) rats before and after FC I/R-injury

\begin{tabular}{llll}
\hline & Con $(\mathbf{n}=10)$ & DM $(\mathbf{n}=10)$ & DMI $(\mathbf{n}=10)$ \\
\hline Body weight (g) & $391 \pm 7$ & $292 \pm 11^{*}$ & $329 \pm 14^{\dagger}$ \\
Before FC I/R-injury & & & \\
Plasma glucose (mg/dl) & $125 \pm 5$ & $460 \pm 15^{*}$ & $153 \pm 10^{\dagger}$ \\
Plasma insulin ( $\mathrm{gg} / \mathrm{L})$ & $2.58 \pm 0.36$ & $0.56 \pm 0.07^{*}$ & $2.04 \pm 0.55^{\dagger}$ \\
After FC I/R-injury & & & \\
Plasma glucose (mg/dl) & $194 \pm 7$ & $508 \pm 43^{*}$ & $350 \pm 51^{\dagger}$ \\
Plasma insulin ( $\mathrm{\mu g} / \mathrm{L})$ & $3.07 \pm 0.36$ & $0.61 \pm 0.10^{*}$ & $1.66 \pm 0.41^{\dagger}$ \\
\hline
\end{tabular}

Data are expressed as mean \pm SEM $\left({ }^{*} P<0.05\right.$ vs. control, ${ }^{\dagger} P<0.05$ vs. DM).

\section{Insulin treatment preserved microvascular functions in} STZ-diabetic rats

The microvascular functions of cremaster muscles were examined by intravital microscopic observation (Figure 1A). Low rates of leukocyte adherence and transmigrating $(1.7 \pm 0.44$ and $1.7 \pm 0.50$ numbers $/ 100 \mu \mathrm{m}$, respectively) were seen in age-matched non-diabetic controls (Figure 1B). In the STZ-diabetic rats, a large increase of adhering and transmigrating $(6.9 \pm 1.26$ and $5.1 \pm 0.85$ numbers $/ 100 \mu \mathrm{m}$, respectively) leukocytes were observed in the postcapillary venules of the cremaster muscle (Figure 1B). By contrast, DMI had a significantly lower number of adhering $(3.1 \pm 0.60$ numbers $/ 100 \mu \mathrm{m})$ and transmigrating $(2.3 \pm 0.46$ numbers $/ 100 \mu \mathrm{m})$ leukocytes in the postcapillary venules of the cremaster muscle (Figure 1B).

\section{Diabetes increased cerebral NO level was corrected by insulin treatment}

The cerebral NO levels were also examined in control, DM, and DMI rats (Figure 2). Under normoxic conditions, the NO level in the left and right cerebral hemispheres was increased in diabetic rats compared with non-diabetic controls (Figure 2A, B). By contrast, insulin treatment reduced $\mathrm{NO}$ levels compared with nontreated diabetic rats (Figure 2A, B). The fold of the NO level in the right to left cerebral hemispheres was no different among the groups (Figure 2C).

Diabetes aggravated FC I/R-induced brain infarction and neurological deficits were rescued by insulin treatment Hyperglycemia and insulin deficiency may have divergent or distinct effects on the progression of ischemic stroke in STZ-diabetic rats. We sought to compare directly the severity of FC I/R-induced infarct volume and neurological deficits (Bederson) in control, diabetic, and insulin-treated diabetic rats. Before FC I/R injury, blood gas parameters $\left(\mathrm{PO}_{2}, \mathrm{PCO}_{2}\right.$, and $\left.\mathrm{pH}\right)$ had been compared and no statistical difference among the groups 


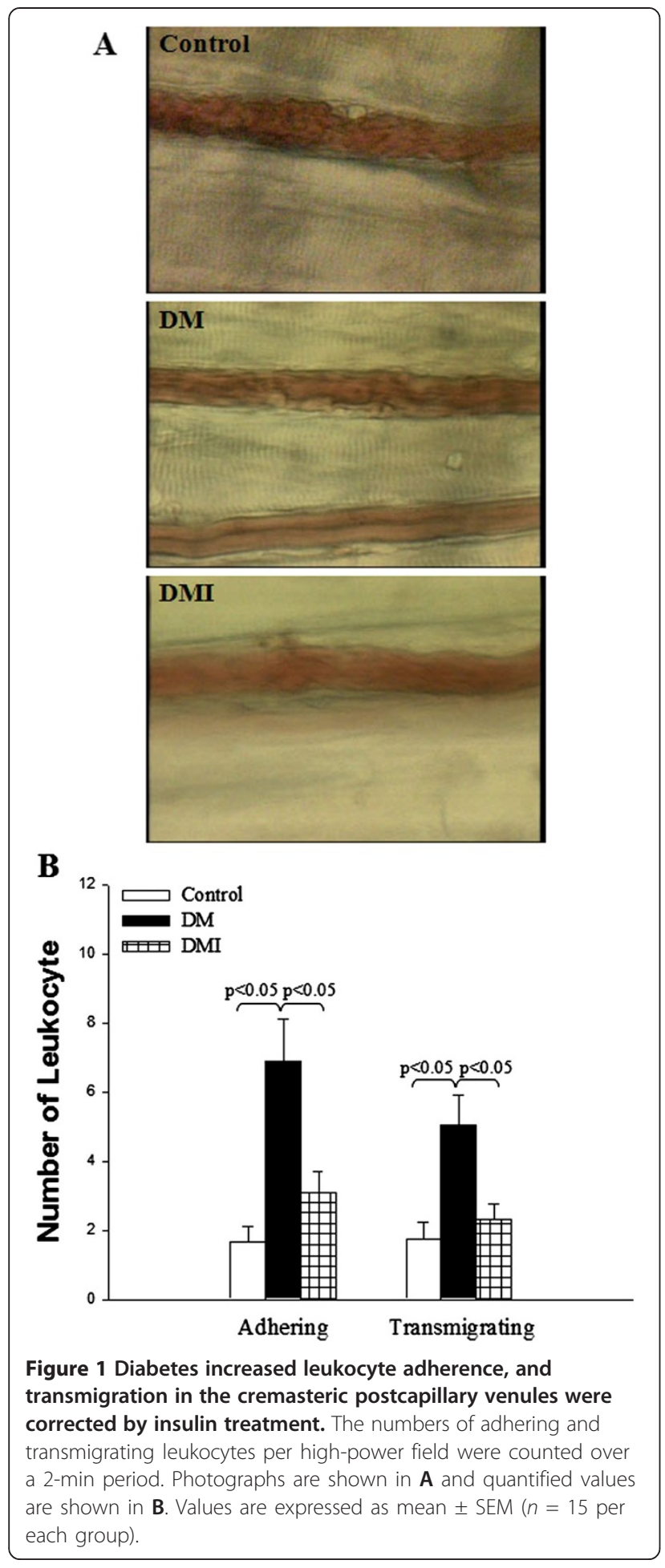

(data not shown) was observed. After FC I/R injury, the infarct volume was $170.12 \pm 14.42 \mathrm{~mm}^{3}$ in the agematched non-diabetic controls $(n=10)$. STZ-diabetic rats indicated a dramatic enhancement of I/R-induced infarct volume by 1.9-fold compared with non-diabetic controls

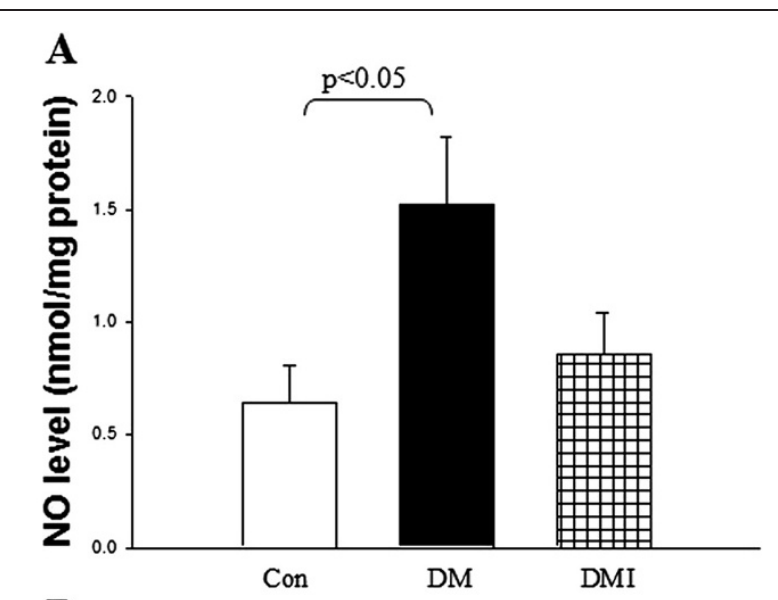

B
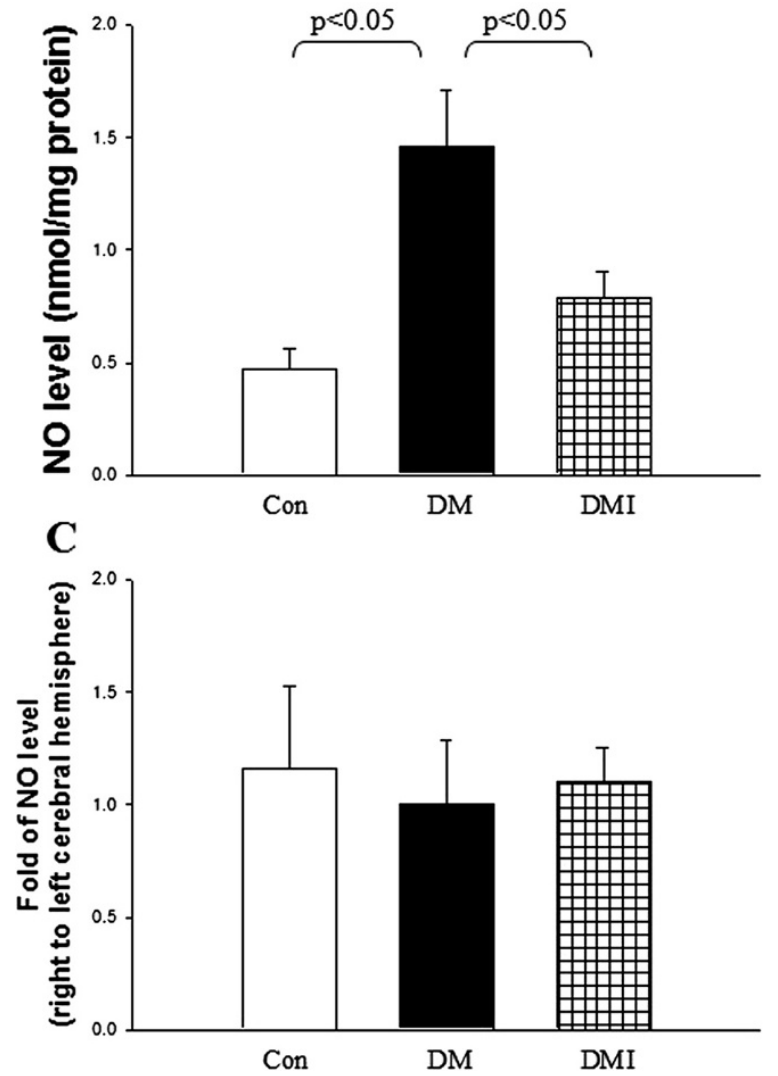

Figure 2 The cerebral NO levels were compared in control, DM, and DMI rats. The left (A) and right (B) cerebral NO levels in rats were evaluated by NO/ozone chemiluminescence technique. (C) The ratio of right to left cerebral hemisphere $\mathrm{NO}$ level was also evaluated among these groups. Results are expressed as mean \pm SEM $(n=7$ to 8 per each group).

$(P<0.001, n=10)$. Consistent with its hypoglycemic effect, insulin treatment significantly reduced I/R-induced infarct volume from $321.79 \pm 29.41$ to $224.91 \pm 22.48 \mathrm{~mm}^{3}$ $(P<0.05, n=10)$ (Figure 3A, B). For evaluation of neurological functions, Bederson tests were performed to 


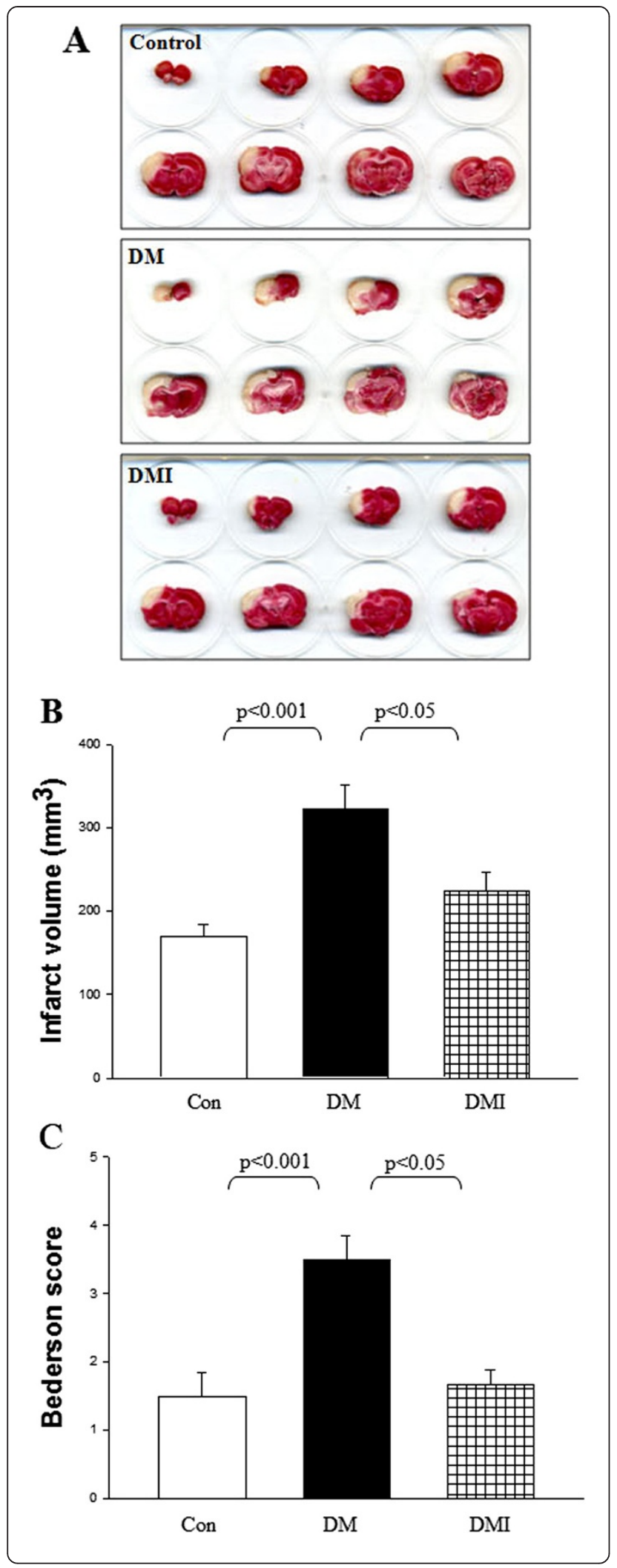

examine the muscle strength and postural reflex, respectively. After ischemic stroke, Bederson scores were $1.5 \pm 0.3$ in non-diabetic control rats (Figure $3 \mathrm{C}$ ). The FC I/Rinduced muscle strength and postural reflex weakness were further impaired in diabetic rats $(3.5 \pm 0.3)$ as compared with non-diabetic controls. By contrast, the diabetic-aggravated neurological deficits were significantly attenuated by insulin treatment $(1.7 \pm 0.2$; Figure 3 C).

General characteristics in Non-diabetic control, STZ-diabetic, and insulin-treated diabetic rats after FC $1 / R$ injury

The characteristics of hyperglycemia and hypoinsulinemia did not influence diabetic rats subjected to FC I/R injury. Plasma glucose and insulin levels in diabetic rats were $508 \pm 43 \mathrm{mg} / \mathrm{dl}$ and $0.61 \pm 0.10 \mu \mathrm{g} / \mathrm{l}$ compared with values of $194 \pm 7 \mathrm{mg} / \mathrm{dl}$ and $3.07 \pm 0.36 \mu \mathrm{g} / \mathrm{l}$ in age-matched nondiabetic controls, respectively $(P<0.001$ and $P<0.05$; Table 1) $24 \mathrm{hr}$ after cerebral infarction. By contrast, insulin treatment significantly attenuated diabetes-induced hyperglycemia $(350 \pm 51$ vs. $508 \pm 43 \mathrm{mg} / \mathrm{dl}, P<0.05)$, and hypoinsulinemia $(1.66 \pm 0.41$ vs. $0.61 \pm 0.10 \mu \mathrm{g} / \mathrm{l}, P<0.01$; Table 1). The plasma triglyceride and cholesterol levels were not significantly different among the groups (data not shown).

\section{Diabetes influenced cerebral NO, superoxide, and peroxinitrite production in $\mathrm{FC} \mathrm{I/R}$ injury was corrected by insulin treatment}

The cerebral NO, superoxide, and peroxinitrite levels were examined in non-diabetic control, STZ-diabetic, and insulin-treated diabetic rats subjected to FC I/R injury (Figure 4). After FC I/R injury, the variability of NO level in the contralateral cerebral hemisphere of the groups (Figure 4A) was consistent with those rats without FC I/R-injury (Figure 2A, B). However, the variability of NO level was altered in the ipsilateral cerebral hemisphere after FC I/R-injury (Figure 4B) compared with non-FC I/R-injured right cerebral hemisphere (Figure 2B). The fold of ipsilateral to contralateral cerebral hemisphere NO level was significantly reduced in FC I/R-injured diabetic rats compared with non-diabetic controls (Figure 4C). By contrast, insulin treatment significantly increased the 

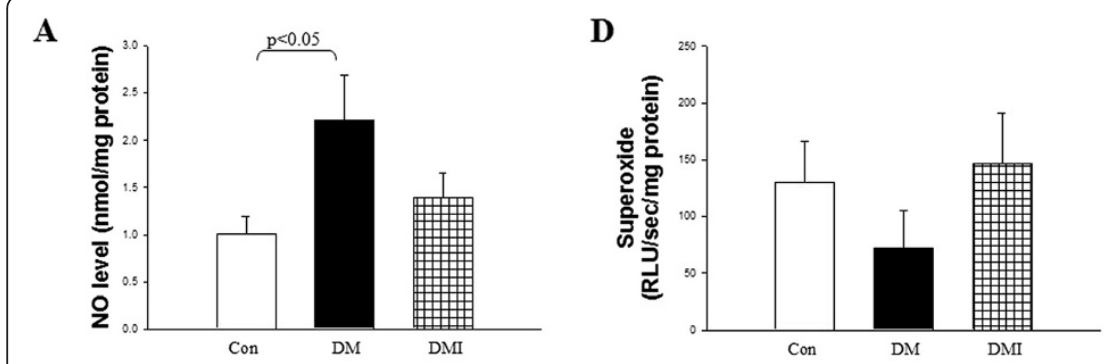

B

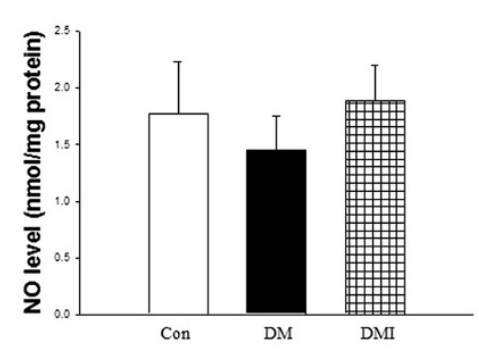

C

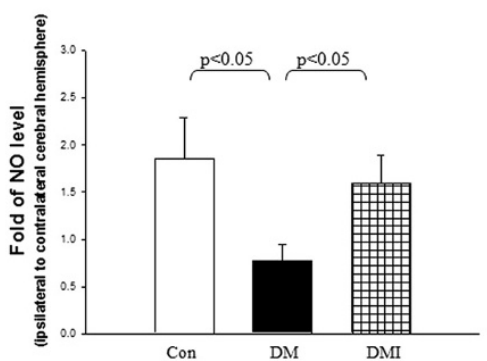

$\mathbf{E}$

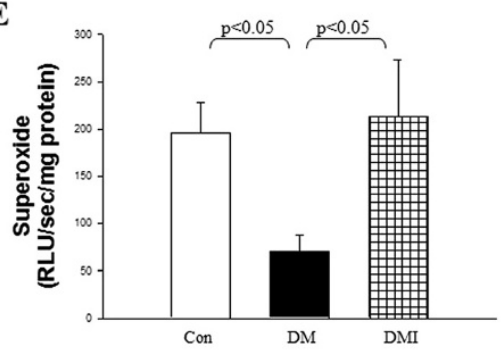

$\mathbf{F}$

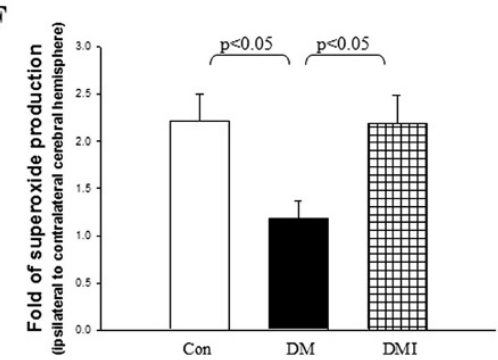

G

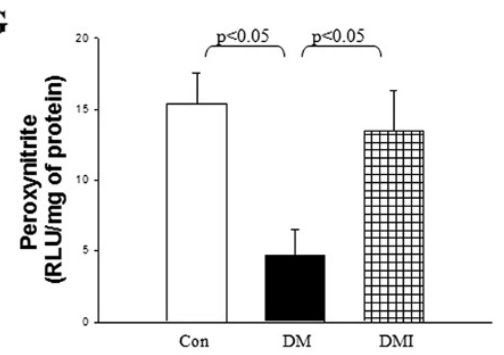

H

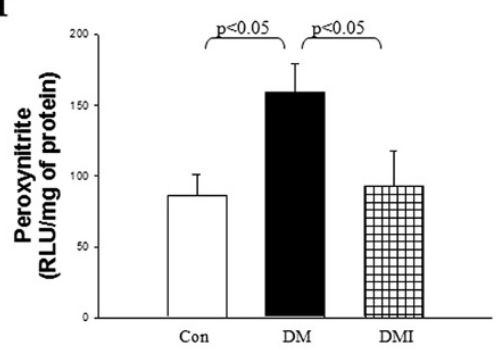

I

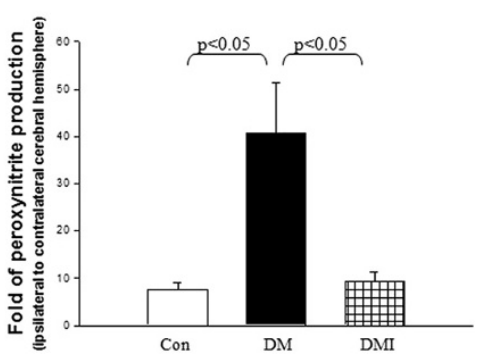

Figure 4 The cerebral NO, superoxide, and peroxynitrite levels in contralateral and ipsilateral cerebral hemisphere were compared in control, DM, and DMI rats after FC I/R injury. The contralateral and ipsilateral cerebral hemisphere NO levels (A and B), superoxide anion contents (D and E), and peroxynitrite production (G and $\mathbf{H})$ were examined in control, DM, and DMI rats with FC I/R injury. The ratio of ipsilateral to contralateral cerebral NO (C), superoxide $\mathbf{( F )}$, and peroxynitrite (I) levels was also evaluated among three groups. Results are expressed as mean $\pm \operatorname{SEM}(n=7$ to 8 per group).

fold of ipsilateral to contralateral cerebral hemisphere NO level (Figure 4C).

Among these three groups, we observed no significant difference in the contralateral cerebral hemisphere superoxide levels after FC I/R injury (Figure 4D). However, superoxide level in the ipsilateral cerebral hemisphere was significantly decreased in diabetic rats compared with agematched controls after FC I/R injury. Insulin treatment significantly reversed diabetes-induced cerebral superoxide level loss (Figure 4E). The fold of ipsilateral to contralateral cerebral hemisphere superoxide level was significantly reduced in FC I/R-injured diabetic rats compared with non-diabetic controls (Figure 4F). The diabetes-induced superoxide level decrease was significantly reversed by insulin treatment (Figure 4F).

After FC I/R injury, in the contralateral cerebral hemisphere, diabetes significantly reduced peroxynitrite levels compared with non-diabetic controls, whereas treatment with insulin showed a significantly higher peroxynitrite levels compared with the non-treated diabetic group
(Figure 4G). By contrast, peroxynitrite level in the ipsilateral cerebral hemisphere was significantly increased in diabetic rats compared with age-matched controls after FC I/R injury. Insulin treatment significantly reversed diabetes-induced cerebral peroxynitrite production (Figure $4 \mathrm{H}$ ). Interestingly, the peroxynitrite level in ipsilateral cerebral hemisphere was 40 -fold to contralateral cerebral hemisphere in STZ-diabetic rats. After FC I/R injury, diabetic rats showed a dramatic enhancement of peroxynitrite production compared with nondiabetic controls, and the diabetes-induced peroxynitrite level increase was significantly attenuated by insulin treatment (Figure 4I).

\section{Discussion and conclusions}

The primary findings of the present study are as follows: (1) Hyperglycemia exacerbated microvascular functions and increased cerebral NO production. (2) Hyperglycemia exacerbated FC I/R-induced infarct volume and neurological dysfunction. (3) Parallel with its hypoglycemic 
effects, insulin also improved microvascular functions and attenuated FC I/R injury in STZ diabetes. (4) Diabetes increased NO reaction with superoxide to form peroxinitrite in the cerebrum after FC I/R injury, and this effect was reversed by insulin treatment.

The present study shows that diabetes increased leukocyte adherence and transmigration in the postcapillary venules of rat cremaster muscle. The cremaster muscle is a powerful model for direct evaluation of microvascular function in vivo. However, the influence of diabetes on leukocyte adherence and transmigration in the postcapillary venules of rat cremaster muscle have yielded conflicting results. Bonnardel-Phu and Vicaut reported that no difference was found in adherent leukocytes or in the leukocyte rolling flux between diabetic and normoglycemic rats [21]. In contrast, Pettersson et al., reported that sustained hyperglycemia results in increased levels of adherent and emigrated leukocytes in mouse models of type 1 and type 2 diabetes [22]. In this study, our results show the impairment of microvascular functions in STZ diabetes. However, the administration of insulin greatly lowered the plasma glucose level, restored the microvascular dysfunction, and rescued FC I/R injury in STZ-diabetic rats.

Oxidative stress, an imbalance between free radical generation and elimination or detoxification, is a principal mediator of cerebral injury during ischemia and reperfusion. In addition, oxidative stress is known to be involved in the development and progression of diabetes and diabetic complications [23]. The unifying pathophysiological mechanism that underlies ischemic stroke in diabetes could be explained by increased production of reactive oxygen species (ROS) and nitrogen species (RNS) [1]. However, studies of diabetic NO levels have yielded conflicting results [5-8]. In this study, we showed that diabetes increased NO production which was reversed by insulin treatment under normoxic conditions. A dominant paradigm to explain the increase of $\mathrm{NO}$ in diabetes is that a $\mathrm{T}$ lymphocyte-dependent autoimmune process activates macrophages or $\beta$-cells [24] result from the decreased production of TGF- $\beta 1$ and increased expression of iNOS pathway by macrophage. In contrast, insulin administration to STZ-induced diabetic rodents decreases macrophage NO production by down-regulation of the iNOS pathway [25]. Evidence suggests that there is an increase in the activity of inducible nitric oxide (iNOS) in diabetes. The increased NO production in diabetic brain with subsequent development of local oxidative stress is supposed to be the possible pathophysiological mechanism to impair endothelium-dependent relaxation of intracranial blood vessels during STZ-induced diabetes [26]. The FC $\mathrm{I} / \mathrm{R}$ injury also increased NO production by 3.7 -fold in non-diabetic control rats. Under normoxic condition, the fold of right to left cerebral hemisphere NO level was not significantly different among the groups. By contrast, the fold of ipsilateral to contralateral cerebral hemisphere NO levels was significantly reduced in FC I/ $\mathrm{R}$-injured diabetic rats and treatment with insulin rescued the above phenomenon. These results indicate that both diabetes and FC I/R injury increased cerebral $\mathrm{NO}$ production, however, diabetes-aggravated FC I/R injuries may be related, with loss of efficacy of the stimulated NO produced. These effects were reversed by insulin treatment.

Furthermore, we found that superoxide level in the ipsilateral cerebral hemisphere and in the fold of ipsilateral to contralateral cerebral hemisphere superoxide levels were significantly decreased in FC I/R-injured diabetic rats compared with non-diabetic controls. The diabetesinduced superoxide level decrease was significantly reversed by insulin treatment. These results indicate that diabetic rats reduced cerebral superoxide levels after FC I/R injury. By contrast, insulin treatment significantly reversed the decrease of superoxide levels.

All ROS and RNS were previously considered toxic agents capable of damaging biomolecules. However, physiological free radicals superoxide and nitric oxide are recently known to be relatively harmless species but are able to mediate or initiate many enzyme- and genedependent reactions in both physiological and pathophysiological processes [27]. NO may react with superoxide to form reactive peroxynitrite (ONOO-). Peroxynitrite is a potent oxidizing agent that can cause DNA fragmentation and lipid peroxidation [28]. Multiple lines of evidence demonstrating the formation of peroxynitrite in diabetic vasculature exist, both in experimental animals and in humans. One of the important pathways of peroxynitrite-mediated vascular dysfunction in diabetes involves activation of the nuclear enzyme poly(ADP-ribose) polymerases (PARP). Peroxynitrite-induced overactivation of PARP consumes $\mathrm{NAD}+$ and consequently ATP culminating in cell dysfunction, apoptosis, or necrosis [29].

Our results show that diabetes slight decreased superoxide anion production but significantly decreased peroxynitrite contents in the contralateral brain region. Many studies demonstrated that endothelium-derived NO was greatly reduced under diabetic state [30,31]. Our previous study also shows that phosphorylated eNOS protein level was dramatic decreased in the brain of FC I/R diabetic rats [32]. Since peroxynitrite is formed by the NOsuperoxide reaction, reduction of superoxide anion and endothelium-derived $\mathrm{NO}$ might be a possible reason for lower peroxynitrite production in the contralateral brain region of $\mathrm{FC} \mathrm{I} / \mathrm{R}$ diabetic rats.

In addition, we also shows that diabetes slight decreased NO level but significantly increased peroxynitrite production in the ipsilateral brain region of FC I/R diabetic rats. Our previous study also shows that phosphorylated eNOS 
protein level was dramatic decreased in brain of FC I/R diabetic rats [32]. This may cause eNOS uncoupling and consequently increase free radical production and decrease endothelium-derived NO. Under this condition, most NO can react with superoxide to form peroxynitrite [33].

In this study, interestingly, although the peroxinitrite level in the contralateral cerebral hemisphere was decreased in diabetic rats compared with that in non-diabetic controls after FC I/R injury and insulin significantly rescued the phenomenon, however, the level of peroxynitrite in the contralateral cerebral hemisphere was very few compared with the amount in the ipsilateral cerebral hemisphere. By contrast, peroxynitrite level in the ipsilateral cerebral hemisphere and in the fold of ipsilateral to contralateral cerebral hemisphere peroxynitrite levels were significantly increased in diabetic rats compared with age-matched controls after FC I/R injury. Insulin treatment significantly reversed the diabetes-induced cerebral peroxynitrite production. Interestingly, the peroxynitrite level in ipsilateral cerebral hemisphere was 40 -fold to contralateral cerebral hemisphere in STZ-diabetic rats. After FC I/R injury, diabetic rats showed a dramatic enhancement of peroxynitrite production compared with non-diabetic controls, and the diabetes-induced peroxynitrite level increase was significantly attenuated by insulin treatment.

These results indicate that diabetes stimulated NO production and descreased superoxide production and peroxynitrite formation in normoxic brain region (contralateral brain area). However, the formation of peroxynitrite in diabetic rats increased significantly in $I / R$ brain region (ipsilateral brain area) which may contribute to the prominent increase in brain injury. The increase in peroxynitrite formation and brain injury can be alleviated by insulin treatment.

In conclusion, glycemic control with insulin treatment may have clinical benefits for stroke patients with diabetes, especially in those with insulinopenic diabetes. Our results show that $\mathrm{NO}$ /superoxide/peroxynitrite formation and its normalization contribute to detrimental and protective effects of diabetes and insulin, respectively, in the context of ischemic stroke. Finally, our findings indicate that insulin renders diabetic rats resistant to acute ischemic stroke by arresting $\mathrm{NO}$ reaction with superoxide to form peroxynitrite.

\section{Competing interests}

The authors declare that they have no competing interests.

\section{Authors' contributions}

LMH, JML and SSH planned experiments, interpreted data and approved the version to be published. LMH, JPH and SSH performed most of the experiments, analyzed data and wrote the manuscript. JPH and SSH participated in the animal surgery. JML, MHY, DEL and YJD participated in acquisition of the study specimens. All authors have read and approved the final manuscript.

\section{Acknowledgments}

This work was supported financially by research grants from Chang Gung Memorial Hospital (CMRPD 180142), Chang Gung University (EMRPD1C0281), Chung Shan Medical University (CSMU-INT-102-06), and the Ministry of Science and Technology (NSC 101-2311-B-182-002 and MOST 103-2320-B-040-001-MY3) of Taiwan to Dr. Li-Man Hung and Dr. Shiang-Suo Huang, respectively.

\section{Author details}

'Department and Graduate Institute of Biomedical Sciences and Healthy Aging Research Center, College of Medicine, Chang Gung University, Tao-Yuan, Taiwan. ${ }^{2}$ Department of Physiology, Chung Shan Medical University and Chung Shan Medical University Hospital, Taichung, Taiwan. ${ }^{3}$ Department of Anesthesiology, Chang Gung Memorial Hospital and Graduate Institute of Clinical Medical Sciences, Chang Gung University, Tao-Yuan, Taiwan. ${ }^{4}$ Department of Pharmacology and Institute of Medicine, Chung Shan Medical University and Department of Pharmacy, Chung Shan Medical University Hospital, No.110, Sec. 1, Jianguo N. Rd, Taichung City 402, Taiwan.

Received: 3 July 2014 Accepted: 2 September 2014

2.

\section{References}

1. Olmez I, Ozyurt H: Reactive oxygen species and ischemic cerebrovascular disease. Neurochem Int 2012, 60:208-212.

2. Malinski T, Bailey F, Zhang ZG, Chopp M: Nitric oxide measured by a porphyrinic microsensor in rat brain after transient middle cerebral artery occlusion. J Cereb Blood Flow Metab 1993, 13:355-358.

3. Fabian $\mathrm{RH}$, Kent TA: Superoxide anion protection during reperfusion is reduced by an antineutrophil antibody after prolonged cerebral ischemia. Free Radic Biol Med 1999, 26:355-361.

4. Maritim AC, Sanders RA, Watkins JB 3rd: Diabetes, oxidative stress, and antioxidants: a review. J Biochem Mol Toxicol 2003, 17:24-38.

5. Calver A, Collier J, Valiance P: Inhibition and stimulation of nitric oxide synthesis in the human forearm arterial bed of patients with insulindependent diabetes. J Clin Invest 1992, 90:2548-2554.

6. Shiekh GA, Ayub T, Khan SN, Dar R, Andrabi Kl: Reduced nitrate level in individuals with hypertension and diabetes. J Cardiovasc Dis Res 2011, 2:172-176.

7. Astaneie F, Afshari M, Mojtahedi A, Mostafalou S, Zamani MJ, Larijani B, Abdollahi M: Total antioxidant capacity and levels of epidermal growth factor and nitric oxide in blood and saliva of insulin-dependent diabetic patients. Arch Med Res 2005, 36:376-381.

8. Ferlito S, Gallina M: Nitrite plasma levels in type 1 and 2 diabetics with and without complications. Panminerva Med 1998, 40:304-308.

9. LeMay DR, Gehua L, Zelenock GB, D'Alecy LG: Insulin administration protects neurologic function in cerebral ischemia in rats. Stroke 1988, 19:1411-1419.

10. Warner DS, Gionet TX, Todd MM, MCAllister AM: Insulin-induced normoglycemia improves ischemic outcome in hyperglycemic rats. Stroke 1992, 23:1775-1780.

11. Szkudelski T: The mechanism of alloxan and streptozotocin action in B cells of the rat pancreas. Physiol Res 2001, 50:537-546.

12. Huang SS, Tsai SK, Chih CL, Chiang LY, Hsieh HM, Teng CM, Tsai MC: Neuroprotective effect of hexasulfobutylated $\mathrm{C} 60$ on rats subjected to focal cerebral ischemia. Free Radic Biol Med 2001, 30:643-649.

13. Tsai SK, Hung LM, Fu YT, Cheng H, Nien MW, Liu HY, Zhang FB, Huang SS: Resveratrol neuroprotective effects during focal cerebral ischemia injury via nitric oxide mechanism in rats. J Vasc Surg 2007, 46:346-353.

14. Bergmeyer HU, Bernt E: Lactate dehydrogenase. UV-assay with Pyruvate and NADH. In Methods of Enzymatic Analysis, Volume 2. New York: Academic Press; 1974:574-579.

15. Bederson JB, Pitts LH, Tsuji M, Nishimura MC, Davis RL, Bartkowski H: Rat middle cerebral artery occlusion: evaluation of the model and development of a neurologic examination. Stroke 1986, 17:472-476.

16. Huang JP, Huang SS, Deng JY, Day YJ, Hung LM: Insulin and resveratrol act synergistically preventing cardiac dysfunction in diabetes but the advantage of resveratrol in diabetics with acute heart attack is antagonized by insulin. Free Radic Biol Me 2010, 49:1710-1721. 
17. Chang CC, Yang MH, Tung HC, Chang CY, Tsai YL, Huang JP, Yen TH, Hung LM: Resveratrol exhibits differential protective effects on fast- and slow-twitch muscles in streptozotocin-induced diabetic rats. J Diabetes 2014, 6:60-67.

18. Lau YS, Tian XY, Huang Y, Murugan D, Achike Fl, Mustafa MR: Boldine protects endothelial function in hyperglycemia-induced oxidative stress through an antioxidant mechanism. Biochem Pharmacol 2013, 85:367-375.

19. Boybeyi Ö, Yazici I, Ünlü G, Aslan MK, Soyer T: Intravital microscopic evaluation of cremasteric microcirculation in experimental testicular torsion. J Pediatr Urol 2013, 9:40-44.

20. Huang SS, Wei FC, Hung LM: Ischemic preconditioning attenuates postischemic leukocyte-endothelial cell interactions: role of nitric oxide and protein kinase C. Cir J 2006, 70:1070-1075.

21. Bonnardel-Phu E, Vicaut E: Reactive oxygen species and acute modulation of albumin microvascular leakage in the microcirculation of diabetic rats in vivo. J Vasc Res 2000, 37:32-38.

22. Pettersson US, Christoffersson G, Massena S, Ahl D, Jansson L, Henriksnäs J: Increased recruitment but impaired function of leukocytes during inflammation in mouse models of type 1 and type 2 diabetes. PLOS One 2011, 6:e22480.

23. Folli F, Corradi D, Fanti P, Davalli A, Paez A, Giaccari A, Perego C, Muscogiuri G: The role of oxidative stress in the pathogenesis of type 2 diabetes mellitus micro-and macrovascular complications: avenues for a mechanistic-based therapeutic approach. Curr Diabetes Rev 2011, 7:313-324.

24. Atkinson MA, Maclaren NK: The pathogenesis of insulin-dependent diabetes mellitus. N Engl J Med 1994, 331:1428.

25. Stevens RB, Sutherland DE, Ansite JD, Saxena M, Rossini TJ, Levay-Young BK, Hering BJ, Mills CD: Insulin down-regulates the inducible nitric oxide synthase pathway: nitric oxide as cause and effect of diabetes? I Immunol 1997, 159:5329-5335.

26. Kitayama J, Faraci FM, Gunnett CA, Heistad DD: Impairment of dilator responses of cerebral arterioles during diabetes mellitus: role of inducible NO synthase. Stroke 2006, 37:2129-2133.

27. Afanas'ev I: Signaling of reactive oxygen and nitrogen species in Diabetes mellitus. Oxid Med Cell Longev 2010, 3:361-373.

28. Pacher P, Beckman JS, Liaudet L: Nitric oxide and peroxynitrite in health and disease. Physiol Rev 2007, 87:315-424.

29. Pacher P, Szabo C: Role of the peroxynitrite-poly(ADP-ribose) polymerase pathway in human disease. Am J Pathol 2008, 173:2-13.

30. Creager MA, Lüscher TF, Cosentino F, Beckman JA: Diabetes and vascular disease: pathophysiology, clinical consequences, and medical therapy: Part I. Circulation 2003, 108:1527-1532.

31. Wu G, Meininger CJ: Impaired arginine metabolism and NO synthesis in coronary endothelial cells of the spontaneously diabetic BB rat. Am J Physiol 1995, 269:H1312-H1318.

32. Huang SS, Lu YJ, Huang JP, Wu YT, Day YJ, Hung LM: The essential role of endothelial nitric oxide synthase activation in insulin-mediated neuroprotection against ischemic stroke in diabetes. J Vasc Surg 2014, 59:483-491.

33. Hink U, Li H, Mollnau H, Oelze M, Matheis E, Hartmann M, Skatchkov M, Thaiss F, Stahl RA, Warnholtz A, Meinertz T, Griendling K, Harrison DG, Forstermann U, Munzel T: Mechanisms underlying endothelial dysfunction in diabetes mellitus. Circ Res 2001, 88:E14-E22.

\section{doi:10.1186/s12929-014-0092-0}

Cite this article as: Hung et al:: Insulin renders diabetic rats resistant to acute ischemic stroke by arresting nitric oxide reaction with superoxide to form peroxynitrite. Journal of Biomedical Science 2014 21:92.

\section{Submit your next manuscript to BioMed Central and take full advantage of:}

- Convenient online submission

- Thorough peer review

- No space constraints or color figure charges

- Immediate publication on acceptance

- Inclusion in PubMed, CAS, Scopus and Google Scholar

- Research which is freely available for redistribution

Submit your manuscript at www.biomedcentral.com/submit 